\title{
Dichloroacetate induces protective autophagy in esophageal squamous carcinoma cells
}

\author{
HONG-YU JIA, HE-NAN WANG, FENG-YU XIA, YAN SUN, HONG-LI LIU, \\ LI-LI YAN, SHAN-SHAN LI, DONG-CHUN JIANG and MEI-MEI XU
}

Department of Digestive Internal Medicine, First Hospital of Qinhuangdao, Hebei 066000, P.R. China

Received January 23, 2017; Accepted June 27, 2017

DOI: $10.3892 / 01.2017 .6562$

\begin{abstract}
Dichloroacetate (DCA) is an inhibitor of pyruvate dehydrogenase kinase, which promotes the flux of carbohydrates into mitochondria and enhances the aerobic oxidation of glucose. DCA has previously been demonstrated to exhibit antitumor properties. The present study revealed that treatment with DCA induced increased levels of autophagy-associated proteins in esophageal squamous carcinoma cells while minimally affecting apoptosis. The present study examined the localization of light chain (LC)-3 by adenovirus infection with a green fluorescent protein (FP)-red FP-LC3 reporter construction and confirmed that DCA treatment induced significant autophagy. Furthermore, the inhibition of DCA-induced autophagy facilitated cell apoptosis and improved the drug sensitivity of esophageal squamous carcinoma cells to DCA and 5-FU (5-fluorouracil). The proliferation of TE-1 cells was markedly inhibited at low concentrations of DCA and 5-FU treatment when subjected to Atg5 mRNA interference, indicating that autophagy performed a protective role in cell survival upon DCA treatment. To determine the underlying mechanism of DCA-induced autophagy, the present study measured alterations in autophagy-associated signaling pathways. Notably, the protein kinase B (Akt)-mechanistic target of rapamycin (mTOR) signaling pathway, an important negative regulator of autophagy, was demonstrated to be suppressed by DCA treatment. These results may direct the development of novel strategies for the treatment of esophageal squamous carcinoma based on the combined use of DCA and autophagy inhibitors.
\end{abstract}

\section{Introduction}

Autophagy is a self-protective cellular mechanism that occurs during nutrient deficiency, which functions to conserve vital

Correspondence to: Dr Mei-Mei Xu, Department of Digestive Internal Medicine, First Hospital of Qinhuangdao, 258 Wenhua Street, Hebei 066000, P.R. China

E-mail: xumeimei007@126.com

Key words: dichloroacetate, autophagy, TE-1, Akt-mTOR components in the cell via degradation and recycling of cytoplasmic constituents (1-4). Furthermore, autophagy may aid cell survival under conditions of starvation, hypoxia, and metabolic and chemotherapeutic stress, but superfluous autophagy may trigger cell death (5). It has been reported that the inhibition of mTOR and PI3K-mTOR can activate autophagy in glioma cells, which may promote glioma cell survival (6). It has also been shown that anticancer drugs that inhibit autophagy are able to promote apoptosis (7-9), which suggests that autophagy has a protective role in tumor cells. This outcome indicates that the role of autophagy acts as a double-edged sword that has the role of both killing and protecting cells.

Dichloroacetate (DCA) is a small inhibitor of pyruvate dehydrogenase kinase (PDK) that activates pyruvate dehydrogenase (PDH) and increases glucose oxidation by promoting the influx of pyruvate into the Krebs cycle. DCA facilitates mitochondrial oxidation of glucose-derived pyruvate to produce ATP. Moreover, it has recently been demonstrated as a promising nontoxic antineoplastic agent that promotes apoptosis in breast, colorectal, and prostate cell carcinoma models (10-13). However, the correlation between DCA and autophagy is unknown, and the mechanism by which DCA induces autophagy is the subject of ongoing investigation. In the present study, we found that DCA induced autophagy in an esophageal squamous carcinoma cell lines, TE-1 and TE- 8 cells. We observed increased expression of light chain (LC)-3B-II and minimal apoptosis following DCA treatment. Additionally, DCA in conjunction with 3-methyladenine (3-MA) to inhibit autophagy significantly enhanced DCA-induced apoptosis. We also observed the inhibition of the Akt-mTOR signaling pathway, a crucial negative regulator of autophagy, which provides insight into the mechanism of DCA-induced autophagy.

\section{Materials and methods}

Cell culture and reagents. Human esophageal squamous carcinoma cells, TE-1, were cultured in RPMI1640 supplemented with $10 \%$ fetal bovine serum and $100 \mathrm{mg} / \mathrm{l}$ streptomycin (Sigma) at $37^{\circ} \mathrm{C}$ in an atmosphere containing $5 \% \mathrm{CO}_{2}$. The reagents used in the present study were DCA, 3-MA (both Sigma-Aldrich, St. Louis, MO, USA), adenovirus (GFP-RFP-LC3; Hanbio Shanghai, China), Lipofectamine 2000 (Invitrogen Life 
Technologies, Sanghai, China), and an MTT kit (Sangon Biotech, Sanghai, China). The antibodies used in the present study were Beclin-1 (Cell Signaling Technology, Inc., MA, USA), LC3-I/II, P62, Atg5, PARP, Pro-caspase-3 (all Abcam, Cambridge, UK), $\beta$-actin (Santa Cruz Biotechnology Inc., Dallas, TX USA), Akt, phospho-Akt, mTOR and p-mTOR (all Cell Signaling Technology,).

Immunofluorescence microscopy. Cells were grown on coverslips in a 24-well plate. Before the experiment, TE-1 cells were infected with GFP-RFP-LC3 adenovirus. At $24 \mathrm{~h}$ post-infection, the media was changed, and the cells were treated with DCA for $24 \mathrm{~h}$. For immunostaining, the cells were fixed in $4 \%$ paraformaldehyde. Cells were incubated with DAPI for 5 min and washed three times with PBS. The coverslips were mounted on slides with Vectashield mounting medium. The images were captured using an LSM 510 Meta confocal microscope (Carl Zeiss MicroImaging $\mathrm{GmbH}$, Jena, Germany) and processed using the software provided by the manufacturer.

Western blot. Proteins were extracted in RIPA buffer $(50 \mathrm{mM}$ Tris-base, 1.0 mM EDTA, $150 \mathrm{mM} \mathrm{NaCl}, 0.1 \%$ SDS, $1 \%$ Triton $\mathrm{X}-100,1 \%$ sodium deoxycholate, and $1 \mathrm{mM}$ PMSF) and quantified using a BCA protein assay kit. Samples were separated using 12\% SDS-PAGE and transferred to PVDF membranes. The membranes were blocked for $1 \mathrm{~h}$ with PBS containing $0.1 \%$ Tween-20 in $5 \%$ skim milk at $37^{\circ} \mathrm{C}$ and subsequently probed by the primary antibodies rabbit-anti-Beclin-1 (diluted 1:1,000), rabbit-anti-Atg5 (diluted 1:1,000), mouse-anti-P62 (diluted 1:1,000), rabbit-anti-LC3 (diluted 1:1,000), rabbit-anti-Procaspase-3 (diluted 1:1,000), mouse-anti-PARP (diluted 1:1,000), rabbit-anti-Akt, mouse-anti-Phospho-Akt, rabbit-anti-mTOR and rabbit-anti-Phospho-mTOR, all used at dilutions of 1:1,000. Blots were incubated with the respective primary antibodies overnight at $4^{\circ} \mathrm{C}$. After washing three times in Tris-buffered saline with Tween-20 $(20 \mathrm{mM}$ Tris- $\mathrm{HCl}$, $150 \mathrm{mM} \mathrm{NaCl}, 0.1 \%$ Tween-20), the blots were incubated with an HRP-conjugated anti-rabbit secondary antibody (diluted 1:5,000) or an HRP-conjugated anti-mouse secondary antibody (diluted 1:5,000) for $1 \mathrm{~h}$ at room temperature. Blots were visualized by western chemiluminescence reagents (Millipore, WBKLS0500). Densitometric quantitation of blotting strips was performed using the Quality-One software. $\beta$-actin was used as an internal control.

Cell viability, proliferation and apoptosis assays. For viability assay, cells were seeded at 5,000 cells per well in 96-well plates overnight at $37^{\circ} \mathrm{C}$. Subsequently, DCA and medium control were added to each well, and the cells were cultured at $37^{\circ} \mathrm{C}$ for the indicated times. Cell viability was estimated using an MTT assay. The absorbance was measured at $570 \mathrm{~nm}$ with a microplate reader (Bio-Rad). Cell proliferation was measured by cell counting. Briefly, cells were re-plated in 24-well plates at a density of $1 \times 10^{5}$ cells/well. Cells were incubated for $24 \mathrm{~h}$, and DCA and medium control were added to each well. Cells were counted at 24 or $48 \mathrm{~h}$ post-treatment with an automated cell counter. Cell apoptosis was analyzed by flow cytometry using propidium iodide staining. All experiments were performed in triplicate.
siRNA preparation and transfection. At g5 knockdown was accomplished by transfecting TE-1 cells with targeting siRNA. Atg5 and control siRNA were synthesized by Sangon Biotech. Cells $\left(3 \times 10^{5}\right)$ were transfected with $1 \mu \mathrm{g}$ siRNA using $2.5 \mu 1$ Lipofectamine 2000 reagent. Scrambled siRNA, which consists of a scrambled sequence that will not lead to specific degradation of any known cellular mRNA, was employed as a negative control.

Statistical analysis. Data are presented as the mean \pm SD. Student's t-test was used for the statistical analysis of the data. All statistical analyses were conducted using SPSS 19.0 (IBM, SPSS, Armonk, NY, USA) statistical software. A P-value of $<0.05$ or $<0.01$ was considered to indicate a statistically significant difference.

\section{Results}

DCA-induced autophagy in TE-1 cells. Autophagy is a protective mechanism against extracellular stress. To investigate the role of DCA in autophagy regulation of TE-1 cell, we examined the localization of LC3 using an immunofluorescence assay. We utilized adenovirus encoding a tandem GFP-RFP-LC3 construct to confirm autophagy induction (14). The basis of this function is that the neutral autophagosome and acidic autolysosome showed different $\mathrm{pH}$ sensitivity. The GFP-LC3 degrades, while the RFP-LC3 maintains the puncta when autophagosome fuses with the lysosome to form autolysosomes. After infection with this adenovirus, we observed the successful introduction of LC3 proteins upon treatment with DCA relative to the control (Fig. 1A-B). Moreover, to examine whether autophagy-related proteins are altered in response to DCA treatment, western blot analysis was performed. The protein levels of LC3-II, P62 (SQSTM-1) and Beclin-1 were examined. We observed a significant decrease in the levels of P62 following DCA treatment relative to control conditions. Moreover, the ratio of the classical autophagy pathway proteins, LC3-II and Beclin-1/ $\beta$-actin, was also markedly augmented (Fig. 1C-D). Taken together, these results indicate that the autophagic flux which occurs in TE-1 cells is enhanced by DCA treatment.

Inhibition of DCA-induced autophagy facilitates apoptosis of TE-1 cells. The induction of autophagy is considered to be a protection behavior against apoptosis in cancer cells (15). To examine the influence of DCA treatment on TE-1 apoptosis, cells were treated with DCA for $24 \mathrm{~h}$, and flow cytometry was performed. Our results show that lower dose DCA treatment did not induce a significant level of apoptosis in TE-1 cells compared with the control. 3-MA, a widely used autophagy inhibitor, was used to investigate the functional role of autophagy in response to DCA treatment. Briefly, TE-1 cells were treated alone or in combination with DCA and 3-MA for $24 \mathrm{~h}$, and the levels of apoptosis were measured. Interestingly, DCA-induced apoptosis was markedly enhanced via combined treatment with 3-MA and DCA compared to either treatment alone or the control group (Fig. 2A-B). Subsequently, the critical apoptosis-related proteins PARP and Pro-caspase- 3 were measured by western blot analysis and were found to be significantly reduced upon treatment with 
A

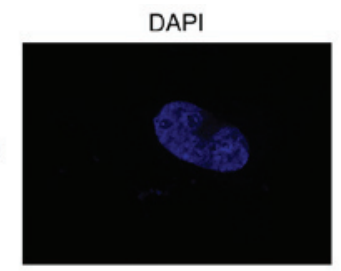

DCA

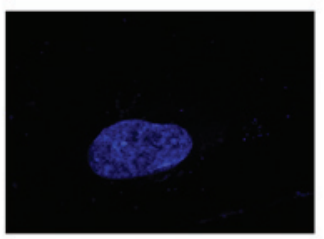

B

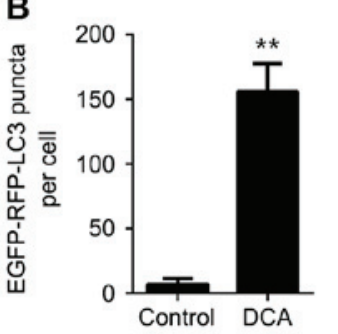

C

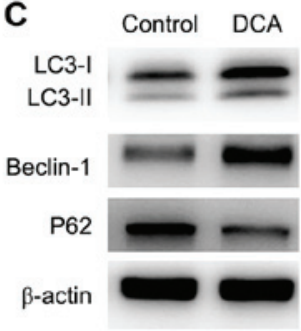

GFP-LC3
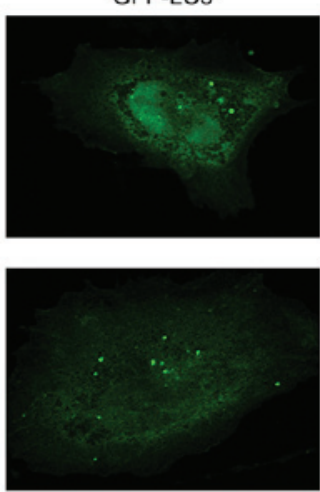
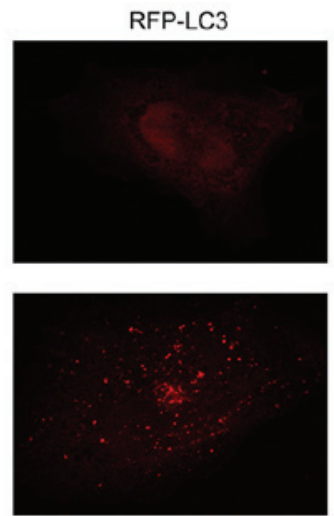
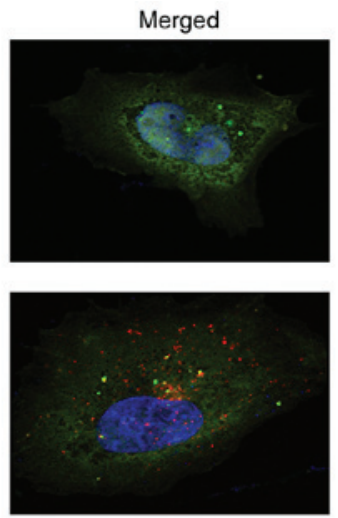

D
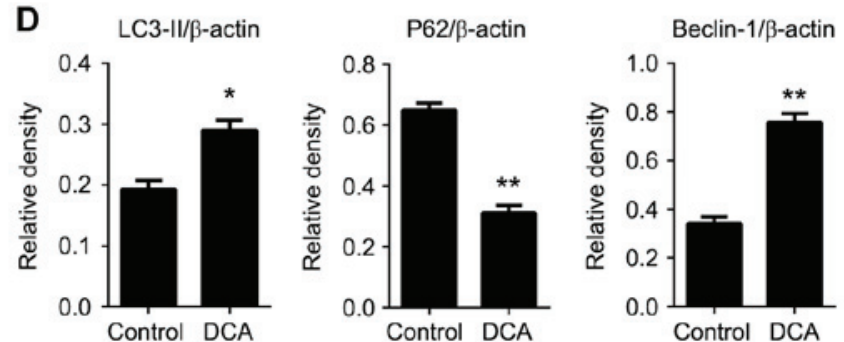

Figure 1. Dichloroacetate (DCA) induces autophagy in TE-1 cells. (A) TE-1 cells infected with GFP-RFP-LC3 adenovirus were treated with 25 mM DCA for $24 \mathrm{~h}$, and the colocalization of EGFP and RFP-LC3 puncta was examined by confocal microscopy. Scale bars, $10 \mathrm{~mm}$. (B) RFP-LC3 puncta were counted using ImageJ software. Data are presented as the mean \pm SD from 3 independent experiments (n.s, not significant, ${ }^{* *} \mathrm{P}<0.01$ vs. control). (C) Cells were treated with DCA (25 mM) for $24 \mathrm{~h}$; the expression of Beclin-1, SQSTM1 and LC3B-II was analyzed by western blot analysis. (D) Comparisons of the intensities of autophagic proteins were statistically estimated and are presented as the mean \pm SD for 3 independent experiments (n.s, not significant; ${ }^{* *} \mathrm{P}<0.01$ vs. control).
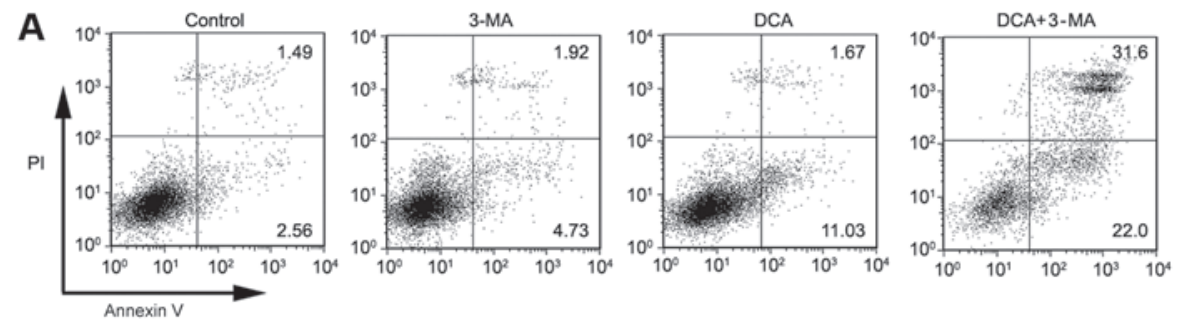

C

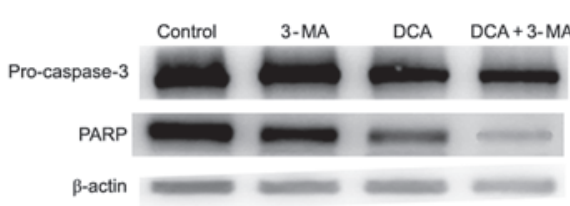

D

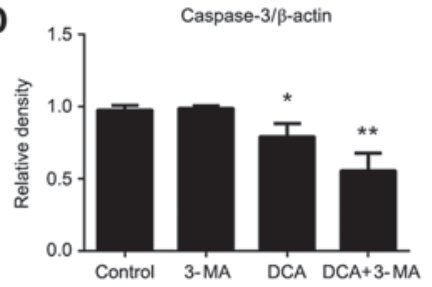

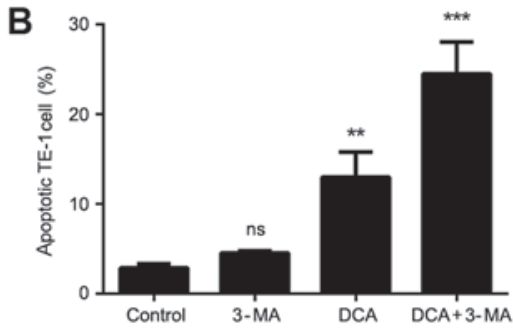

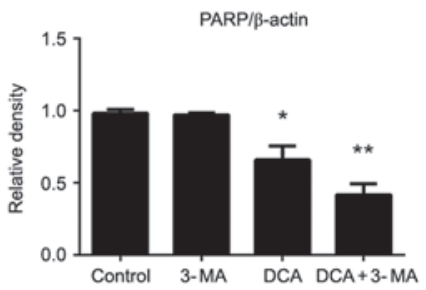

Figure 2. Inhibition of autophagy enhances DCA-induced apoptosis in TE-1 cells. (A, B) Cells were cotreated with or without $25 \mathrm{mM}$ DCA and $5 \mathrm{mM} 3$-MA for $24 \mathrm{~h}$, and apoptosis was determined by Annexin V-FITC/PI staining and flow cytometry (mean \pm SD for 3 independent experiments; ${ }^{* *} \mathrm{P}<0.01$ compared with control or DCA and 3-MA treatment alone). (C) Cells were cotreated with or without $25 \mathrm{mM}$ DCA and 3-MA for $24 \mathrm{~h}$; the expression of PARP, Pro-caspase-3 was analyzed by western blot. (D) Comparisons of the intensities of apoptosis-related proteins were statistically estimated and are presented as the mean \pm SD for 3 independent experiments (n.s, not significant; ** $\mathrm{P}<0.01$ ).

DCA and 3-MA (Fig. 2C-D). These results indicated that the antitumor effect of DCA might be strengthened when the level of autophagy in tumor cells is inhibited.

RNA interference of Atg5 enhanced the drug sensitivity of $D C A$ and 5-fluorouracil (5-FU). 5-fluorouracil (5-FU) is a commonly used antitumor chemotherapeutic, and combination treatment with DCA may achieve better outcomes (16).
Induction of autophagy in tumor cells is considered one of the primary mechanisms of chemoresistance (17). To investigate whether the inhibition of autophagy could improve the effects of combination chemotherapy, we used Atg5 siRNA to inhibit autophagy. The interference of Atg5 mRNA was confirmed by western blot (Fig. 3A and B). As shown in Fig. 3C-D, cell number and viability were significantly reduced in cells knocked down for Atg5 in the DCA- and 

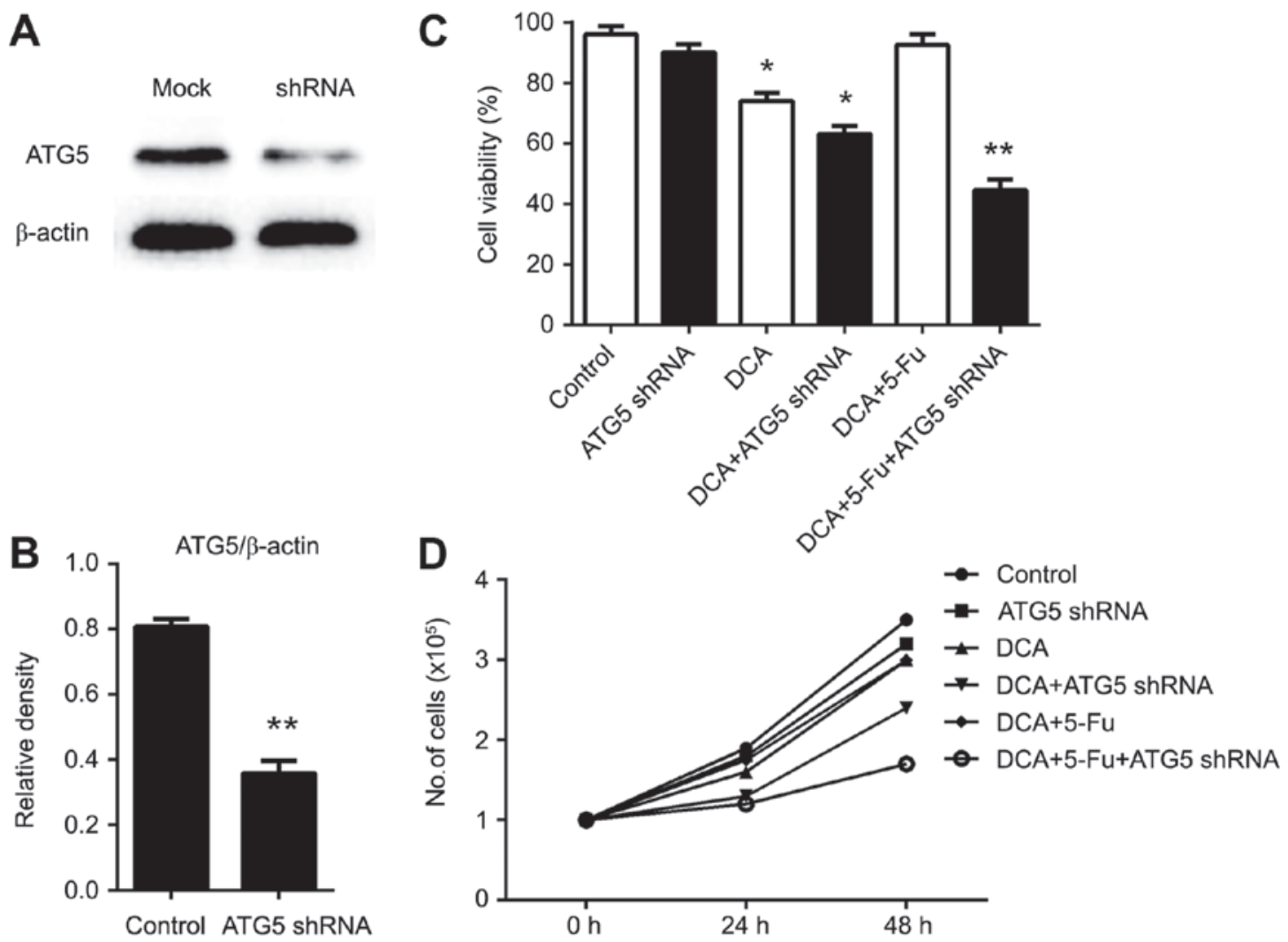

Figure 3. Knockdown of autophagy enhanced drug sensitivity of DCA and 5-fluorouracil (5-FU) in TE-1 cells. (A-B) Knockdown of ATG5 was confirmed and analyzed by western blot analysis. Comparisons of the intensities of ATG5 proteins were statistically estimated and represented as the mean \pm SD for 3 independent experiments (n.s, not significant; ${ }^{* *} \mathrm{P}<0.01$ vs. control). (C) TE-1 cells were infected with ATG5-knockdown plasmid for $12 \mathrm{~h}$, then treated with DCA or $5 \mu \mathrm{g} / \mathrm{ml} 5$-FU for $24 \mathrm{~h}$, and MTT assays were performed to assess cell proliferation. (D) TE-1 cells were infected with ATG5-knockdown plasmid for $12 \mathrm{~h}$, then treated with DCA or $5 \mu \mathrm{g} / \mathrm{ml} 5$-FU for 24 or $48 \mathrm{~h}$, and cell proliferation was analyzed by cell counting. Data are presented as the mean \pm SD for 3 independent experiments. ${ }^{* *} \mathrm{P}<0.01 ; \mathrm{ns}$, not significant.

5-FU-treated conditions. In addition, apoptosis was markedly increased in cells treated with the DCA and 5-FU combination treatment, indicating that inhibition of Atg5 expression could enhance drug sensitivity.

DCA-induced autophagy occurs via inhibition of the AKT-mTOR signaling pathway. It is known that the AKT/mTOR pathway is a key negative regulator of autophagy (17). Here, we examined whether DCA treatment is involved in the regulation of the Akt-mTOR pathway. The phosphorylation status of both Akt and mTOR was significantly decreased in DCA-treated cells compared with the control. These findings suggested that DCA induced autophagy by inhibition of the AKT/mTOR pathway in TE-1 cells (Fig. 4A-B). To investigate whether DCA played an identical role in other cells, we examined the proliferation and the level of autophagy-related proteins in relation to DCA treatment in TE- 8 cells, which correspond to moderately differentiated esophageal squamous carcinoma. The cell autophagic activity was increased during DCA treatment, and the cell viability was comparable to that of TE-1 cells and was inhibited significantly upon treatment with DCA, 5-FU and ATG5 shRNA. To gain insight into the mechanism of DCA-induced autophagic activity in TE-8 cells, we examined the AKT-mTOR signaling pathway by western blot assay. Our results revealed that DCA treatment enhanced the dephosphorylation of AKT and mTOR, which indicated that
DCA-induced autophagy through the AKT-mTOR signaling pathway was pervasive in tumor cells (Fig. 4C-E).

\section{Discussion}

DCA, as an inhibitor of the 3-PDPK1-4 family, has been reported to inhibit tumor proliferation by reversing the bio-energetic profile in a variety of cancer models (18-20). DCA inhibits the activity of pyruvate dehydrogenase kinase (PDK) and subsequently increases the activity of pyruvate $\mathrm{PDH}$, which reverses mitochondrial dysfunction and reactivates mitochondria-dependent apoptosis $(10,19,21)$. Autophagy is a self-protective cellular mechanism that provides energy for cells. Previous studies have demonstrated that protective autophagy induced by chemotherapy and radiotherapy can resist cell apoptosis $(8,9)$. However, the internal interaction between autophagy and DCA was unknown in esophageal squamous carcinoma cells.

In the present study, we found that DCA induced autophagy in human esophageal squamous carcinoma cells with minimal apoptosis and induced high levels of autophagy-related proteins. Moreover, autophagy inhibition by Atg 5 siRNA or 3-MA treatment significantly improved DCA-induced apoptosis and drug sensitivity in TE-1 cells. These results suggest that autophagy plays a protective role in tumor cells in response to DCA treatment. Hence, low-concentration DCA 
A
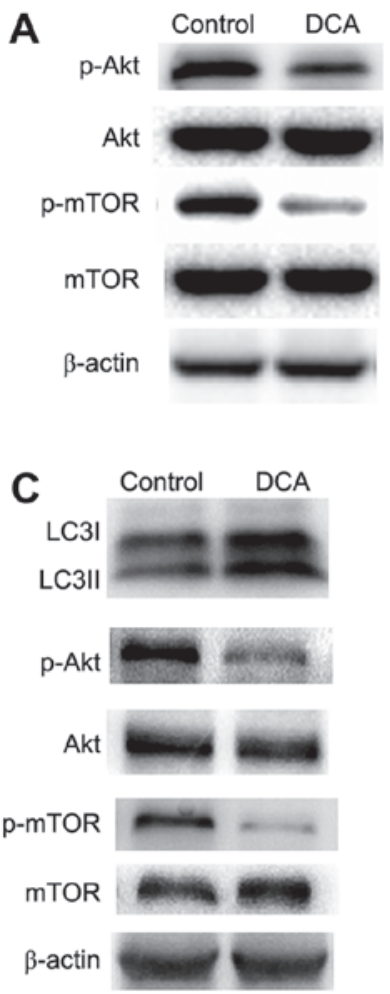

B

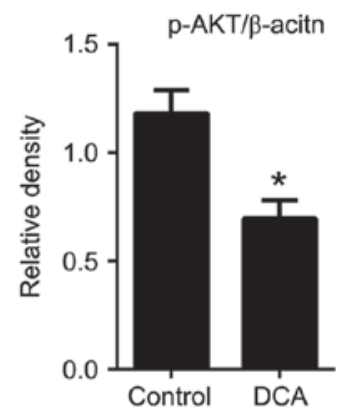

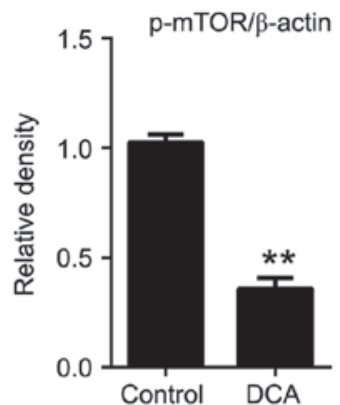

D
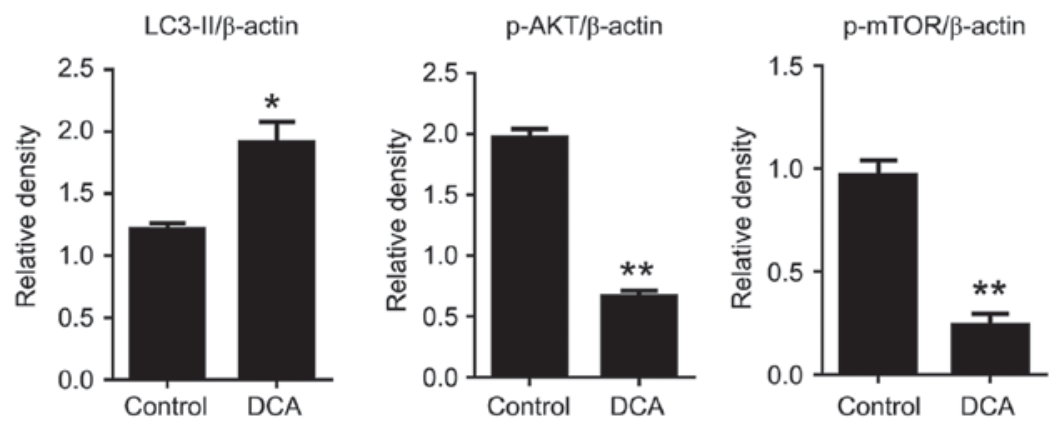

\section{E}

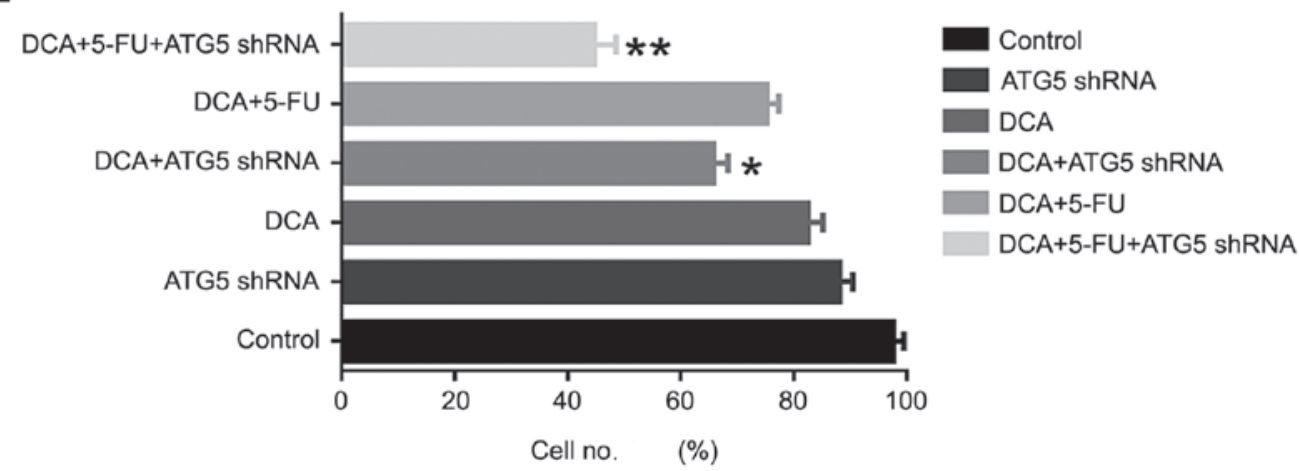

Figure 4. Akt-mTOR pathway was involved in DCA-induced autophagy in TE-1 cells. (A) Cells were treated with DCA ( $25 \mathrm{mM})$ for $24 \mathrm{~h}$; the expression of Akt, p-Akt, mTOR, p-mTOR was analyzed by western blot. (B) Comparisons of the intensities of proteins were statistically estimated and are presented as the mean \pm SD for 3 independent experiments (ns, not significant; * $\mathrm{P}<0.01$ vs. control). (C) TE-8 cells were treated with DCA ( $25 \mathrm{mM})$ for $24 \mathrm{~h}$; the expression of LC3, Akt, p-Akt, mTOR, p-mTOR was analyzed by western blot analysis. (D) Comparisons of the intensities of autophagic proteins were statistically estimated and are presented as the mean $\pm \mathrm{SD}$ for 3 independent experiments (n.s, not significant; ${ }^{* *} \mathrm{P}<0.01 \mathrm{vs}$. control). (E) TE- 8 cells were infected with ATG5-knockdown plasmid for $12 \mathrm{~h}$, then treated with DCA or $5 \mu \mathrm{g} / \mathrm{ml} 5-\mathrm{FU}$ for $24 \mathrm{~h}$, and cell proliferation was analyzed. Data are represented as the mean \pm SD for 3 independent experiments. ${ }^{* *} \mathrm{P}<0.01$; ns, not significant.

treatment in conjunction with autophagy inhibitors may exert antitumor activity.

The Akt-mTOR signaling is an important negative pathway that regulates autophagy. In the present study, we demonstrated that the phosphorylation of Akt and mTOR was inhibited by DCA treatment, indicating that DCA-induced autophagy occurs by inhibition of Akt-mTOR signaling. Lin et al reported that DCA induced autophagy was accompanied by ROS production and mTOR inhibition, reduced lactate excretion, reduced $\mathrm{k}_{\mathrm{PL}}$ and increased $\mathrm{NAD}^{+} / \mathrm{NADH}$ ratio in colorectal carcinoma cells, which was consistent with our findings (18).

In conclusion, DCA inhibits glycolysis and reduces lactate accumulation, which destroys the acidified tumor microenvironment. DCA treatment resulted in significant apoptosis in human colon cancer cells, but apoptosis was decreased upon treatment with DCA under hypoxia (22), which may suggest that a high level of autophagy occurs in hypoxic conditions. In the present study, we found that DCA treatment increased autophagy-related protein production and inhibited the mTOR pathway in esophageal squamous carcinoma cancer cells. These results demonstrate that autophagy plays a protective role in DCA treatment, and further studies are required to verify these results under hypoxia.

\section{References}

1. Mizushima N, Levine B, Cuervo AM and Klionsky DJ: Autophagy fights disease through cellular self-digestion. Nature 451: 1069-1075, 2008. 
2. Gupta VK, Scheunemann L, Eisenberg T, Mertel S, Bhukel A, Koemans TS, Kramer JM, Liu KS, Schroeder S, Stunnenberg HG, et al: Restoring polyamines protects from age-induced memory impairment in an autophagy-dependent manner. Nat Neurosci 16: 1453-1460, 2013.

3. Denton D, Nicolson S and Kumar S: Cell death by autophagy: Facts and apparent artifacts. Cell Death Differ 19: 87-95, 2012.

4. Teng RJ, Du J, Welak S, Guan T, Eis A, Shi Y and Konduri GG: Cross talk between NADPH oxidase and autophagy in pulmonary artery endothelial cells with intrauterine persistent pulmonary hypertension. Am J Physiol Lung Cell Mol Physiol 302: L651-L663, 2012.

5. Baehrecke EH: Autophagy: Dual roles in life and death? Nat Rev Mol Cell Biol 6: 505-510, 2005.

6. Fan QW and Weiss WA: Autophagy and Akt promote survival in glioma. Autophagy 7: 536-538, 2011.

7. Wang K, Liu R, Li J, Mao J, Lei Y, Wu J, Zeng J, Zhang T, Wu H, Chen L, et al: Quercetin induces protective autophagy in gastric cancer cells: Involvement of Akt-mTOR- and hypoxia-induced factor $1 \alpha$-mediated signaling. Autophagy 7: 966-978, 2011.

8. Cheng P, Ni Z, Dai X, Wang B, Ding W, Rae Smith A, Xu L, Wu D, He F and Lian J: The novel BH-3 mimetic apogossypolone induces Beclin-1- and ROS-mediated autophagy in human hepatocellular carcinoma [corrected] cells. Cell Death Dis 4: e489, 2013.

9. Lin JF, Tsai TF, Liao PC, Lin YH, Lin YC, Chen HE, Chou KY and Hwang TI: Benzyl isothiocyanate induces protective autophagy in human prostate cancer cells via inhibition of mTOR signaling. Carcinogenesis 34: 406-414, 2013.

10. Bonnet S, Archer SL, Allalunis-Turner J, Haromy A, Beaulieu C, Thompson R, Lee CT, Lopaschuk GD, Puttagunta L, Bonnet $\mathrm{S}$, et al: A mitochondria- $\mathrm{K}+$ channel axis is suppressed in cancer and its normalization promotes apoptosis and inhibits cancer growth. Cancer Cell 11: 37-51, 2007.

11. Cao W, Yacoub S, Shiverick KT, Namiki K, Sakai Y, Porvasnik S, Urbanek C and Rosser CJ: Dichloroacetate (DCA) sensitizes both wild-type and over expressing Bcl-2 prostate cancer cells in vitro to radiation. Prostate 68: 1223-1231, 2008.
12. Sun RC, Fadia M, Dahlstrom JE, Parish CR, Board PG and Blackburn AC: Reversal of the glycolytic phenotype by dichloroacetate inhibits metastatic breast cancer cell growth in vitro and in vivo. Breast Cancer Res Treat 120: 253-260, 2010.

13. Sun W, Zhou S, Chang SS, McFate T, Verma A and Califano JA: Mitochondrial mutations contribute to HIF1alpha accumulation via increased reactive oxygen species and up-regulated pyruvate dehydrogenease kinase 2 in head and neck squamous cell carcinoma. Clin Cancer Res 15: 476-484, 2009.

14. Yuan K, Huang C, Fox J, Laturnus D, Carlson E, Zhang B, Yin Q, $\mathrm{Gao} \mathrm{H}$ and $\mathrm{Wu} \mathrm{M}$ : Autophagy plays an essential role in the clearance of Pseudomonas aeruginosa by alveolar macrophages. J Cell Sci 125: 507-515, 2012.

15. Jiang $X$, Overholtzer $M$ and Thompson CB: Autophagy in cellular metabolism and cancer. J Clin Invest 125: 47-54, 2015.

16. Tong J, Xie G, He J, Li J, Pan F and Liang H: Synergistic antitumor effect of dichloroacetate in combination with 5-fluorouracil in colorectal cancer. J Biomed Biotechnol 2011: 740564, 2011.

17. Rubinsztein DC, Codogno P and Levine B: Autophagy modulation as a potential therapeutic target for diverse diseases. Nat Rev Drug Discov 11: 709-730, 2012.

18. Lin G, Hill DK, Andrejeva G, Boult JK, Troy H, Fong AC, Orton MR, Panek R, Parkes HG, Jafar M, et al: Dichloroacetate induces autophagy in colorectal cancer cells and tumours. Br J Cancer 111: 375-385, 2014.

19. Michelakis ED, Webster L and Mackey JR: Dichloroacetate (DCA) as a potential metabolic-targeting therapy for cancer. Br J Cancer 99: 989-994, 2008.

20. Papandreou I, Goliasova T and Denko NC: Anticancer drugs that target metabolism: Is dichloroacetate the new paradigm? Int J Cancer 128: 1001-1008, 2011.

21. Michelakis ED, Sutendra G, Dromparis P, Webster L, Haromy A, Niven E, Maguire C, Gammer TL, Mackey JR, Fulton D, et al: Metabolic modulation of glioblastoma with dichloroacetate. Sci Transl Med 2: 31ra34, 2010.

22. Shahrzad S, Lacombe K, Adamcic U, Minhas K and Coomber BL: Sodium dichloroacetate (DCA) reduces apoptosis in colorectal tumor hypoxia. Cancer Lett 297: 75-83, 2010. 